\title{
Integrated Flexible Dynamic Maneuver Loads Models based on Aerodynamic Influence Coefficients of a 3D Panel Method
}

\author{
Thiemo M. Kier * \\ German Aerospace Center (DLR), Institute of System Dynamics and Control, Oberpfaffenhofen, Germany
}

\begin{abstract}
The integration of loads analysis models using so called aerodynamic influence coefficients (AICs) is described. These AICs relate a change of normal velocity at panel control points to a change in panel pressure distribution, allowing to consider aeroelastic effects in a straight forward manner. The aerodynamic method employed for aeroelastic applications is typically the Vortex or Doublet Lattice Method, discretizing mean lifting surfaces. In this paper, the AICs are obtained by a 3D panel method, which significantly increases the geometric fidelity and accounts for previously unmodeled effects. This paper demonstrates the application for manoeuvre loads analysis using a quasi-steady assumption. Two test cases are presented: a steady pull-up trim case and a transient roll manoeuvre. The results using the AIC approach are validated by comparison with steady computations of the 3D panel method.
\end{abstract}

Keywords: integrated flexible flight dynamics model, maneuver loads analysis, 3D Panel Methods, Aerodynamic Influence Coefficients

\section{Nomenclature}

$M_{\infty}$ Mach number

$a_{\infty} \quad$ speed of sound

$U_{\infty}$ free stream velocity

$q_{\infty} \quad$ dynamic pressure

$c_{\text {ref }}$ reference chord length

$\omega$ natural frequency

$k$ reduced frequency

$\boldsymbol{\Phi}_{S} \quad$ steady velocity potential

$\boldsymbol{\Phi}_{U} \quad$ unsteady velocity potential

$\mathbf{w}_{j}$ downwash vector vector

$\mathbf{Q}_{j j} \quad$ AIC matrix

P load vector

u displacement vector

$\boldsymbol{\Phi}_{g h}$ structural modal (eigenvector) matrix

$\mathbf{T}_{k g}$ spline matrix interconnecting structural and aerodynamic degrees of freedom

Subscripts

$g \quad$ structural degree of freedom set

$b \quad$ rigid body degree of freedom set

$f \quad$ modal flexible degree of freedom set

$h \quad$ combined modal (rigid body + flexible) degree of freedom set

$j \quad$ aerodynamic control point degree of freedom set

$k \quad$ aerodynamic panel reference point degree of freedom set

$x 1$ aerodynamic rigid body states ( $\alpha, \beta$, normalized roll-, pitch- and yaw-rate)

$x 2$ aerodynamic control surface degree of freedom set

\footnotetext{
*Team Lead Flight Dynamics and Loads, Department of Aircraft Systems Dynamics, Thiemo.Kier@dlr.de
} 


\section{Introduction}

For the certification of a new aircraft type, it has to be demonstrated that its structure can withstand the loads acting on it without damage. In order to design the structure accordingly, a so called loads envelope is computed. To determine this envelope, loads analysis models need to be simulated many times in the entire flight envelope at different air speeds, altitudes, mass cases and for different types of manoeuvres and gusts. Further, these models must account for the flexibility of the airframe and the resulting change in the distributed aerodynamic loading in order to determine the shear force, bending and torsion moment along the axes of the individual aircraft components. To reduce the amount of simulations, often prior experience and engineering judgement is applied. Despite this effort the number of required load cases can still easily exceed the 100.000s.

Hence, loads analysis models need to be fast to simulate, but also sufficiently accurate, to cover this enormous amount of simulations. Clearly, for this type of application, the use of Computational Fluid Dynamics (CFD) coupled with Computational Structural Mechanics (CSM) is still too expensive, in spite of the impressive advances in recent computing technology. Therefore, much faster aerodynamic methods are essential to handle the large number of simulations required for loads analysis. Typically, for these kind of aeroelastic applications, the aerodynamic methods are based on linear potential theory, which solve the (unsteady) Prandtl-Glauert equation. Traditionally, lifting surfaces methods such as the Vortex Lattice Method (VLM) [1], respectively its unsteady counterpart the Doublet Lattice Method (DLM) [2, 3, 4] are used. Since lifting surface methods discretize the mean surface of aerodynamic surfaces, merely the pressure difference between upper and lower surface is calculated.

In this paper, the 3D panel method NEWPAN [5] from Flow Solutions Ltd. is used to discretize the actual surface. The increased geometric fidelity enables to compute surface pressures of volumetric bodies. The use of a 3D panel method also allows to account for effects, such as e.g. flight mechanical yaw-roll coupling. In most lifting surface schemes, these effects are not present, due to early linearization and simplifying assumptions in their derivations.

When the surface pressures are calculated by higher fidelity aerodynamic methods like Euler and RANS CFD solvers, the coupling with the structural dynamics and flight mechanics is usually done by co-simulation[6, 7]. The pressure loads and deflections are passed over between the aerodynamics code and the equation of motion of the flexible body, e.g. using predictor-corrector schemes. To reduce the simulation time, faster aerodynamic methods such as the previously mentioned panel methods can be used. However, still co-simulation strategies are often employed in this case $[8,9]$.

The approach in the present paper, is to obtain a linear relationship about a reference flight state between the normal velocities at the control points $\mathbf{w}_{\mathbf{j}}$ and the perturbation surface pressures coefficients $\mathbf{c}_{\mathbf{p}}$ of the individual panels, the so called Aerodynamic Influence Coefficient (AIC) matrix $\mathbf{Q}_{j j}$. The 3D Panel Method NEWPAN allows to output such AIC matrices as a function of a harmonic excitation. The resulting AICs are fully populated, complex valued matrices of size $n_{\text {panels }}$ by $n_{\text {panels }}$. They need to be setup only once in a preprocessing step and can then be reused in different loads analysis scenarios. In the AIC approach the pressures are obtained by a simple matrix multiplication, instead of recomputing the pressures at each time step for the changing flow conditions. Further, this relationship allows to express the problem in a closed form, without having to resort to co-simulation. A fit of the unsteady transfer functions the so called Rational Function Approximation (RFA) [10,11], allows to express the problem as a system of nonlinear ordinary differential equations (ODE), which can be integrated in time using Runge-Kutta schemes. This approach is in line with classical aeroelasticity [12] using the VLM/DLM, but extending its fidelity and nonlinear aspects.

The present paper is concerned with using the described AIC approach for manoeuvre loads analysis. First, loads analysis model integration in general is discussed and the necessary equations are formulated. Then, the governing equations for the $3 \mathrm{D}$ panel method and corresponding boundary conditions are presented. Next, the requirements for manoeuvre loads analysis and the implications of a quasi-steady approximation for the aerodynamics are discussed. The implementation details of the aerodynamic model based on 3D panel method AICs are outlined. Finally, the results are validated by comparing the present AIC approach to regular 3D panel method computations for selected manoeuvres.

\section{Loads Analysis Model Integration}

The model integration and simulation has been implemented in the loads analysis environment VarLoads [13, $14,15,16,11,17,18]$. The following section describes the general principles regarding the integration aspects of the loads analysis model, i.e. the structural model, the equations of motion and the external forcing due to propulsion and the aerodynamics. The model equations in VarLoads are expressed in closed from, in contrast to co-simulation schemes in other simulation environments, i.e. similarly to classical aeroelasticity the gradient information of the AIC matrices is used. Furthermore, the equations are formulated as nonlinear system of ODEs to be amendable to time domain integration. 


\section{A. Structural Dynamics, Equations of Motion and Load Recovery}

The starting point, when setting up the equations of motion for a loads analysis model for a flexible aircraft is an Finite Element Model (FEM). This FEM usually consists of 100.000s of degrees of freedom (DoFs). Static condensation can be used to reduce the problem size by several orders of magnitude. The method employed is known as the Guyan reduction[19], where condensation points $(g-s e t)$ are placed along a loads reference axis. The mass distributions are prepared for the corresponding payload/fuel cases and connected to the $g-$ set. Subsequently a modal analysis is carried out and only part of the modal basis is retained to further reduce the model size and computational cost.

The eigenvalues and eigenvectors define the generalized coordinates of the $h-$ set. The zero eigenvalues represent the rigid body motion. The $h-$ set can be partitioned into six rigid body DoFs $(b-s e t)$ and flexible part $(f-s e t)$. The rigid body mode shapes $\boldsymbol{\Phi}_{g b}$ and the retained modes of the eigenvector matrix $\boldsymbol{\Phi}_{g f}$ are used to generalized the equations of motion, which are given in the frequency domain by

$$
\left\{-\omega^{2}\left[\begin{array}{cc}
\mathbf{M}_{b b} & \mathbf{0} \\
\mathbf{0} & \mathbf{M}_{f f}
\end{array}\right]+\mathrm{j} \omega\left[\begin{array}{cc}
\mathbf{0} & \mathbf{0} \\
\mathbf{0} & \mathbf{B}_{f f}
\end{array}\right]+\left[\begin{array}{cc}
\mathbf{0} & \mathbf{0} \\
\mathbf{0} & \mathbf{K}_{f f}
\end{array}\right]\right\}\left[\begin{array}{l}
\mathbf{u}_{b} \\
\mathbf{u}_{f}
\end{array}\right]=\left[\begin{array}{c}
\boldsymbol{\Phi}_{g b}^{\mathrm{T}} \\
\mathbf{\Phi}_{g f}^{\mathrm{T}}
\end{array}\right] \mathbf{P}_{g}^{e x t}(\omega) .
$$

Note that the rigid body $b-$ set DoFs in (1) are defined in a earth fixed coordinate frame.

A suitable set of equations of motion to account for large rigid body motions and linear flexibility is derived in the references[20,21, 22, 23]. The nonlinear equations of motion describe the movement relative to a "mean axes" body reference frame. Equations of motion for an unrestrained flexible aircraft accounting for large rigid body motions are given by

$$
\begin{aligned}
{\left[\begin{array}{c}
\mathbf{m}_{b}\left(\dot{\mathbf{V}}_{b}+\boldsymbol{\Omega}_{b} \times \mathbf{V}_{b}-\mathbf{T}_{b E} \mathbf{g}_{E}\right) \\
\mathbf{J}_{b} \dot{\boldsymbol{\Omega}}_{b}+\boldsymbol{\Omega}_{b} \times\left(\mathbf{J}_{b} \boldsymbol{\Omega}_{b}\right)
\end{array}\right]=\boldsymbol{\Phi}_{g b}^{\mathrm{T}} \mathbf{P}_{g}^{\text {ext }}(t) } \\
\mathbf{M}_{f f} \ddot{\mathbf{u}}_{f}+\mathbf{B}_{f f} \dot{\mathbf{u}}_{f}+\mathbf{K}_{f f} \mathbf{u}_{f}=\boldsymbol{\Phi}_{g f}^{\mathrm{T}} \mathbf{P}_{g}^{\text {ext }}(t),
\end{aligned}
$$

where $\boldsymbol{\Phi}_{g b}$ is the rigid body modal matrix about the center of gravity and in directions as customary in flight mechanics, i.e x-forward, z-down. $\mathbf{V}_{b}$ and $\boldsymbol{\Omega}_{b}$ are the velocity, respectively angular velocity vectors in the body frame of reference. The matrix $\mathbf{T}_{b E}$ transforms the gravitational vector from an earth fixed $(E)$ to the body fixed coordinate frame $(b)$ as a function of Euler angles.

In order to recover the nodal loads $\mathbf{P}_{g}$ for a subsequent sizing of the structure, the force summation method (FSM) [12] is employed. Thus, subtraction of the inertial loads $\mathbf{P}_{g}^{\text {iner }}$ from the external loads, yields

$$
\mathbf{P}_{g}=\mathbf{P}_{g}^{\mathrm{ext}}-\underbrace{\mathbf{M}_{g g}\left\{\boldsymbol{\Phi}_{g b} \ddot{\mathbf{u}}_{b}+\boldsymbol{\Phi}_{g f} \ddot{\mathbf{u}}_{f}\right\}}_{\mathbf{P}_{g}^{\text {iner }}}
$$

In the case of the nonlinear equations of motion (2), the rigid body acceleration is given as

$$
\ddot{\mathbf{u}}_{b}=\left[\begin{array}{c}
\dot{\mathbf{V}}_{b}+\boldsymbol{\Omega} \times \mathbf{V}_{b}-\mathbf{T}_{b E} \mathbf{g}_{E} \\
\dot{\boldsymbol{\Omega}}_{b}+\mathbf{J}_{b}^{-1}\left(\boldsymbol{\Omega}_{b} \times\left(\mathbf{J}_{b} \boldsymbol{\Omega}_{b}\right)\right)
\end{array}\right] .
$$

The FSM requires the external forces to be available on the structural grid. This allows to accounts for the static part directly on the physical grid, and therefore has a good convergence behavior. Also, the FSM allows to discern between the contributions of inertial and aerodynamic forces, which might be insightful for design decisions. Then cut loads can be computed by integrating the nodal loads along the loads reference axes of each aircraft component. The envelope of the cut loads is used as sorting criteria to obtain the critical load cases used for the structural sizing.

\section{B. External Forces: Aerodynamics and Propulsion}

With the equations of motion defined, the external forces have to be determined. The major sources of these external forces are the propulsion and the aerodynamics. The propulsion forces are simply modeled as concentrated forces at the engine locations, accounting for thrust as well as gyroscopic loads. The other major contribution to the excitation forces $\mathbf{P}_{g}^{\text {ext }}$ stem from the aerodynamics.

In aeroelastic applications the aerodynamic pressures are determined by using a linear relationship between the normalwash at the control point to the panel pressure, i.e. a change of the flow, normal to the panel at control point results in a change in pressure distribution. This allows to easily account for flexible deformation, which are simply treated as change in the normalwash vector $\mathbf{w}_{j}$. The pressure coefficients are computed by

$$
\Delta \mathbf{c}_{\mathbf{p}_{j}}=\mathbf{Q}_{j j} \mathbf{w}_{j},
$$


where $\mathbf{Q}_{j j}$ is the so called AIC matrix. Traditionally, the Vortex Lattice and the Doublet Lattice Methods are used to obtain these AIC matrices. The Doublet Lattice Method provides the complex valued AIC matrix as function of reduced frequency $k=\frac{c_{r e f} / 2}{U_{\infty}} \omega$, which are in essence the unsteady aerodynamic transfer functions. In frequency domain calculations, cf. equation (1) the complex AICs can be used directly. For time domain simulations, a Rational Function Approximation (RFA) [10,11], is required to transform the AICs to the Laplace domain. The rational functions can then be cast in form of a system of linear ordinary differential equation amendable to time integration.

The load transformation to panel reference point is done by integrating the pressures, which is mostly a simple multiplication with the aerodynamic box area. In some classical aerodynamic panel methods additional moments occur due to an offset between control point and pressure application point, cf. [24]. These are accounted for by introducing rotational degrees of freedom in the aerodynamic panel $(k-s e t)$ and the respective moment arms into the integration matrix $\mathbf{S}_{k j}$. Multiplication with the dynamic pressure yields the aerodynamic forces.

$$
\mathbf{P}_{k}^{\text {aero }}=q_{\infty} \mathbf{S}_{k j} \mathbf{c}_{\mathbf{p}_{j}}
$$

Next, the boundary condition for the normalwash has to be considered:

$$
\mathbf{w}_{j}(k)=\left(\mathbf{D}^{\mathbf{x}}{ }_{j k}+\frac{\mathrm{d}}{\mathrm{d} t}\left(\frac{c_{r e f} / 2}{U_{\infty}}\right) \cdot \mathbf{D}^{\mathbf{t}}{ }_{j k}\right) \mathbf{u}_{k}(t),
$$

where the matrix $\mathbf{D}^{\mathbf{x}}{ }_{j k}$ accounts for a change in downwash due to a change of the normal vector with respect to the free stream direction and the matrix $\mathbf{D}^{\mathbf{t}}{ }_{j k}$ for additional downwash due to movement of the boundary in direction of the panel normal. The factor $\frac{c_{r e f} / 2}{U_{\infty}}$ in equation (7) is needed due to the conversion from reduced to natural frequency. The vector $\mathbf{u}_{k}(t)$ represents the motion of the aerodynamic reference points.

When the nonlinear equations of motion (2) are used, special attention to the boundary condition is required. The vectors $\mathbf{V}_{b}$ and $\boldsymbol{\Omega}_{b}$ are defined in a body carried frame of reference. Hence, the steady deflection of rigid body modes does not induce aerodynamic loads. Therefore, the differentiation matrix $\mathbf{D}^{\mathbf{x}}$ needs to be canceled for the rigid body modes. Otherwise, this would imply that the orientation (Euler angles) of the aircraft would directly result in additional aerodynamic forces. The cancelation of the differentiation matrix can also be regarded as a coordinate transformation from an earth fixed to a body fixed coordinate system.

Finally, the aerodynamic loads have to be mapped to the structural degrees of freedom. The matrix connecting the displacements of the the structural grid $(g-s e t)$ to the aerodynamic grid $(k-s e t)$ is called spline matrix $\mathbf{T}_{k g}$.

$$
\mathbf{u}_{k}=\mathbf{T}_{k g} \mathbf{u}_{g}
$$

This mapping is achieved, e.g. by employing radial basis functions, such as the commonly used Infinite Plate Spline (IPS) [25], or by using beam splines [26]. The physical interpretation is that the structure behaves plate or beam like and that the respective degrees of freedom sets $(k-s e t$ and $(g-s e t))$ lie on the same structure described by the spline basis functions. The aerodynamic loads can be mapped back onto the structure with the transpose of the spline matrix, based on the principal of virtual work.

$$
\mathbf{P}_{g}^{\text {aero }}=\mathbf{T}_{k g}^{\mathrm{T}} \mathbf{P}_{k}^{\text {aero }}
$$

Similarly, the modal matrix $\boldsymbol{\Phi}_{g f}$, and its transpose connect the flexible part of the equations of motion (1) and (2) to the aerodynamic model.

\section{3D Panel Method}

Instead of using the mean lifting surface discretization of the Vortex, respectively Doublet Lattice Method, a three dimensional panel method is used to model the flow about the actual surface. The panel method NEWPAN [5] from Flow Solutions Limited calculates the steady mean flow pressures, as well as the complex perturbation pressures due to harmonic excitation on the surface, discretized by quadrilateral or triangular panels. Further, NEWPAN is also able to output complex AIC matrices, which allow for a direct integration in the previously described modeling scheme.

The derivation of the governing flow equations, which the panel method the solves, proceeds in two stages. In the first stage, the steady flow about the mean surface is associated with the steady velocity potential $\Phi_{S}$. The governing equation is given by the steady Prandtl-Glauert equation:

$$
\left(1-M_{\infty}^{2}\right) \frac{\partial^{2} \Phi_{S}}{\partial x^{2}}+\frac{\partial^{2} \Phi_{S}}{\partial y^{2}}+\frac{\partial^{2} \Phi_{S}}{\partial z^{2}}=0
$$

Using a Göthert Type 2 transformation [27], the problem can be reduced to a Laplace type equation $\nabla^{2} \Phi_{S}=0$. 
This steady solution about a, possibly deformed reference shape, defines the flight state about which an unsteady linearization is performed. The unsteady solution is then found by solving the linearized frequency domain variant of the unsteady Prandtl-Glauert equation

$$
\left(1-M_{\infty}^{2}\right) \frac{\partial^{2} \Phi_{U}}{\partial x^{2}}+\frac{\partial^{2} \Phi_{U}}{\partial y^{2}}+\frac{\partial^{2} \Phi_{U}}{\partial z^{2}}-2 \mathrm{j} \omega \frac{M_{\infty}}{a_{\infty}} \frac{\partial \Phi_{U}}{\partial x}-\left(\frac{\omega^{2}}{a_{\infty}^{2}}\right) \Phi_{U}=0,
$$

where the unsteady potential is given as $\mathrm{e}^{\mathrm{j} \omega t} \Phi_{U}(x, y, z)$. This equation can be converted to a Helmholtz type equation

$$
\nabla^{2} \Phi_{U}+\kappa^{2} \Phi_{U}=0, \quad \text { with } \quad \kappa=k \frac{M_{\infty}}{1-M_{\infty}^{2}},
$$

to determine the solution, where $\kappa$ is a frequency parameter depending on the reduced frequency and Mach number. The total velocity potential is then $\Phi(x, y, z, \omega)=\Phi_{S}(x, y, z)+\mathrm{e}^{\mathrm{j} \omega t} \Phi_{U}(x, y, z)$, from which the complex unsteady pressure is calculated via a linearized version of the unsteady Bernoulli equation.

To obtain the steady mean flow from NEWPAN, the flight state is defined by the Mach number $M_{\infty}$, the angle of attack $\alpha$ and sideslip $\beta$, as well as the roll, pitch and yaw rates ( $p, q$ and $r$ ). Further the control surface deflections are specified, which are accounted for by a finite rotation of the corresponding normal vectors about a given hingeline.

The unsteady flow is then calculated as a function of reduced frequency about this steady flight state in a small perturbation sense. By default the complex pressures for the rigid body and control surface modes are computed. Additionally, structural modes can be defined as displacement vectors of the nodes of the surface panel mesh. For the unsteady flow calculation the AIC matrix can be obtained for specified reduced frequencies and used for external computation of the pressures. The AIC matrix for $k=0$ is then real valued and will be used for the quasi-steady approximation in the manoeuver loads analysis.

Some differences and direct ramifications of using 3D panel method versus the commonly used DLM should be noted. The DLM also solves the unsteady Prandtl-Glauert equation (11), however $\Phi_{U}$ represents the acceleration (or pressure) potential rather than the velocity potential. Otherwise, the governing equation are formally equivalent, i.e. the same type of elementary solutions, such as doublets and sources are valid. The DLM directly yields the pressure difference, hence no pressure recovery via the Bernoulli equation is required. However, an integration has to be performed to obtain the velocities, which are required to satisfy the flow tangency condition at the control point. Another direct result from using the acceleration potential is the absence of a modeled wake. Since there is no pressure jump across the wake, the DLM solution implicitly accounts for a flat wake in free stream direction. This can be a big relief to the modeling effort, however such a flat wake might be a crude approximation to a force free wake. Velocity potential methods allow for a relaxation to ensure the wake is force free. This comes at the cost that the sometimes tedious modeling of the wake is required.

Further, when thick bodies are modeled in potential flow, additional pressure contributions arise. These are not associated with the normalwash, but with tangential flow at the panels. Therefore, the 3D Panel Method, requires the additional motion induced body terms $\mathbf{D} \mathbf{P}^{\mathbf{x}}{ }_{j k}$ and $\mathbf{D P}^{\mathbf{t}}{ }_{j k}$.

$$
\Delta \mathbf{c}_{\mathbf{p}_{j}}(k)=\mathbf{Q}_{j j}\left(\mathbf{D}^{\mathbf{x}}{ }_{j k}+\mathrm{j} k \cdot \mathbf{D}^{\mathbf{t}}{ }_{j k}\right) \mathbf{u}_{k}(k)+\left(\mathbf{D P}^{\mathbf{x}}{ }_{j k}+\mathrm{j} k \cdot \mathbf{D P}^{\mathbf{t}}{ }_{j k}\right) \mathbf{u}_{k}(k)
$$

Due to linearization about a flat reference geometry in the DLM, these terms are not required. Further, in the DLM any coupling between the steady and unsteady flow is lost, which is accounted for by the thick body panel discretization.

\section{Manoeuvre Loads Simulation}

Flight loads analysis models can be classified into two categories, manoeuver loads and gust loads models. Models for manoeuvre loads analysis are based on nonlinear rigid body equations of motion to account for large amplitude responses as a result from prescribed pilot inputs. This necessitates an aerodynamic database, covering the nonlinearities in the flight regime of interest. Models for gust loads analysis on the other hand, only consider small perturbations around a trimmed flight state. In this case, both structural dynamics as well as unsteady aerodynamic effects are important. The unsteady aerodynamic increments are usually considered to be linear. In [11] an approach to unify these models has been presented, where on top of the manoeuvre analysis aspects, the unsteady aerodynamics are modeled as an additional effect by means of a RFA of the frequency domain transfer function. This unified modeling approach allowed the separation of the quasi-steady from the unsteady aerodynamic effects.

Compared to a gust load case, the change of normal-wash at the control points for a maneuvering aircraft can be considered slow, i.e. the spectral content of the excitation is limited to low frequencies. In this case the quasi steady assumption holds, i.e. that there is neither a change in magnitude and nor a phase shift in the aerodynamic transfer 
functions over the low frequency range. E.g. in unsteady incompressible 2D airfoil aerodynamics this implies that the Theodorsen function [28] is constant over the reduced frequency range with its value for $k=0$, i.e. a value of one. Equivalently, for the aircraft geometry this means that the AIC matrix at a reduced frequency of zero can be used to compute the pressures for all low excitation frequencies.

This is in line with the general approach for flight mechanical simulations. However, one notable unsteady addition is commonly incorporated: the lag in downwash from the wing to the empennage is modeled by use of the $\dot{\alpha}$-derivatives and air speed dependent time delays. The major difference between flight mechanical models and manoeuver loads models is the necessity in loads analysis to account for distributed aerodynamic forces over the entire airframe, instead of just computing the aircraft total force and moment coefficients to feed the rigid body equations of motion. This results in a significant increase of the modeling effort, which is usually accounted for by sacrificing some level of detail concerning the nonlinearities in the aerodynamic database.

The manoeuvres that have to be simulated for loads analysis are specified in the CS 25 subpart C [29]. These so called design manoeuvres include, e.g. symmetrical pull up and push over manoeuvres (CS 25.331), accelerated and steady roll manoeuvres (CS 25.349), one sided engine failures (CS 25.367), or yawing manoeuvres (CS 25.351). Some of these can be covered by trim calculations as e.g. the symmetrical $2.5 \mathrm{~g}$ pull-up, others need to be simulated in time, particularly when interaction with flight control laws or pilot response needs to be considered.

\section{Implementation using Aerodynamic Influence Coefficients}

The following section describes a proof of concept implementation of using the aerodynamic influence coefficients of the 3D panel method Newpan to compute the aerodynamic forces. The loads analysis models are based on the quasi-steady assumption explained in the previous section. Additionally, a simple interpolation scheme is employed to consider larger variations of the flight state to meet the general requirements for a manoeuvre loads simulation.

Therefore, linearization at two distinct points at $\alpha=0 \mathrm{deg}$ and $\alpha=10 \mathrm{deg}$ are chosen to account for nonlinearities associated with the angle of attack. At these points the AIC matrices $\mathbf{Q}_{j j}(\alpha)$ for $k=0$, the relevant differentiation matrices of eq. (13) and the mean flow steady pressures $c_{p 0}$ are computed.

During time integration with a higher order Runge-Kutta schemes, the model is evaluated many times per time step. Therefore, to shorten the simulation times, merely the generalized aerodynamic forces due to the inputs that are driving the equations of motion (2) are computed. The required inputs that induce aerodynamic loads are given by the rigid body velocities $\mathbf{V}_{b}$ and rates $\Omega_{b}$ (abbreviated by $\dot{\mathbf{u}}_{b}$ ), the control surface deflections denoted by $\mathbf{u}_{x 2}$, the flexible modal displacements $\mathbf{u}_{f}$ and the corresponding flexible modal velocities $\dot{\mathbf{u}}_{f}$. Instead of directly using the rigid body velocities $\dot{\mathbf{u}}_{b}$ defined about the center of gravity, the typical quantities about an aerodynamic reference point, namely $\alpha, \beta$ and roll pitch and yaw rates normalized by free stream velocity and reference length $\frac{U_{\infty}}{c_{r e f}}$ are computed. These quantities are assembled in an aerodynamic state vector and denoted $\mathbf{u}_{x 1}$.

The steady pressure coefficients $\mathbf{c}_{\mathbf{p}_{0}}(\alpha)$ at the linearization points are generalized to yield directly the input for the equations of motion. The contribution due to the angle of attack can then be interpolated directly as a function of angle of attack.

$$
\mathbf{Q}_{h 0}(\alpha)=\boldsymbol{\Phi}_{g h}^{\mathrm{T}} \mathbf{T}_{k g}^{\mathrm{T}} \mathbf{S}_{k j} \mathbf{c}_{\mathbf{p}_{0}}(\alpha)
$$

Similarly, the gradient information of the AIC matrices is expressed in generalized influence coefficient matrices at each linearization point. Analogously to the generalized steady values in eq. (14), the matrices are interpolated as function of angle of attack, during the simulation. Required are the effects due to rigid body aerodynamic states,

$$
\mathbf{Q}_{h x 1}(\alpha)=\mathbf{\Phi}_{g h}^{\mathrm{T}} \mathbf{T}_{k g}^{\mathrm{T}} \mathbf{S}_{k j}\left[\mathbf{Q}_{j j}(\alpha) \mathbf{D}^{\mathbf{t}}{ }_{j k}(\alpha)+\mathbf{D P}^{\mathbf{t}}{ }_{j k}(\alpha)\right] \mathbf{\Phi}_{k x 1}
$$

due to control surface deflections,

$$
\mathbf{Q}_{h x 2}(\alpha)=\boldsymbol{\Phi}_{g h}^{\mathrm{T}} \mathbf{T}_{k g}^{\mathrm{T}} \mathbf{S}_{k j}\left[\mathbf{Q}_{j j}(\alpha) \mathbf{D}^{\mathbf{x}}{ }_{j k}(\alpha)+\mathbf{D P}^{\mathbf{x}}{ }_{j k}(\alpha)\right] \mathbf{\Phi}_{k x 2}
$$

due to modal displacements,

$$
\mathbf{Q}_{h f}(\alpha)=\boldsymbol{\Phi}_{g h}^{\mathrm{T}} \mathbf{T}_{k g}^{\mathrm{T}} \mathbf{S}_{k j}\left[\mathbf{Q}_{j j}(\alpha) \mathbf{D}^{\mathbf{x}}{ }_{j k}(\alpha)+\mathbf{D P}^{\mathbf{x}}{ }_{j k}(\alpha)\right] \mathbf{T}_{k g} \boldsymbol{\Phi}_{g f}
$$

and due to modal velocities

$$
\mathbf{D Q}_{h f}(\alpha)=\boldsymbol{\Phi}_{g h}^{\mathrm{T}} \mathbf{T}_{k g}^{\mathrm{T}} \mathbf{S}_{k j}\left[\mathbf{Q}_{j j}(\alpha) \mathbf{D}^{\mathbf{t}}{ }_{j k}(\alpha)+\mathbf{D P}^{\mathbf{t}}{ }_{j k}(\alpha)\right] \mathbf{T}_{k g} \boldsymbol{\Phi}_{g f} .
$$

These AIC matrices (15)-(14) can be set up in a model initialization phase and are then interpolated linearly as function of $\alpha$ during the simulation. This way, the sizes of the matrices are kept as small as possible, allowing for 
rapid simulation times. Thus, the vector of generalized aerodynamic forces driving the equations of motion is:

$$
\mathbf{P}_{h}^{\text {aero }}(\alpha)=q_{\infty}\left[\mathbf{Q}_{h x 1}(\alpha) \cdot \mathbf{u}_{x 1}+\mathbf{Q}_{h x 2}(\alpha) \cdot \mathbf{u}_{x 2}+\mathbf{Q}_{h f}(\alpha) \cdot \mathbf{u}_{f}+\mathbf{D Q}_{h f}(\alpha) \cdot \frac{U_{\infty}}{\left(c_{r e f} / 2\right)} \dot{\mathbf{u}}_{f}+\mathbf{Q}_{h 0}(\alpha)\right]
$$

Pressures and physical structural forces, required for the loads recovery are calculated in a postprocessing step based on the trajectory generated by the simulation. Note that the contribution to the aerodynamic forces from the angle of attack, $\mathbf{Q}_{h 0}$, are directly interpolated. Hence, the gradient $\mathbf{Q}_{h x 1}$ wrt. $\alpha$ needs to be set to zero. Some further simplifications are applied due to the fact that this is a proof of concept implementation. Firstly, the thick body terms $\mathbf{D P}^{\mathbf{x}}{ }_{j k}$ and $\mathbf{D P}^{\mathbf{t}}{ }_{j k}$ were neglected. For thin wings the error is small, however for thick bodies the influence of these terms is considerable. Secondly, the rates of control surface deflection have been set to zero. As the control surface deflection is not a state variable in the ODE sense, the calculation of the rate would have to be performed by finite differencing during simulation. The effects were deemed small enough to be negligible.

\section{Results and Validation of the Present Approach}

For the simulation, a model of a large passenger aircraft with two engines was chosen. The aerodynamic panel model is depicted in 1 and consists of circa 15000 panels. Eight individual control surfaces are defined, a pair of elevators, inner and outer ailerons, the horizontal tailplane and a rudder. These control surfaces are combined for three pilot control inputs to initiate roll, pitch and yaw commands, the horizontal stabilizer is used for longitudinal trimming. The structural model is described by 90 flexible and 6 rigid body modes.

The AIC matrices in the described implementation scheme have 186 inputs ( 5 rigid body aerodynamic states, 3 pilot inputs, 90 modal displacements and 90 modal velocities) and 96 outputs ( 6 rigid body forces and moments and 90 flexible generalized aerodynamic forces). This is the size of the matrix that needs to be linearly interpolated as a function of alpha during the simulation and multiplied with the input vector. The resulting output vector is multiplied with the dynamic pressure $q_{\infty}$ to yield the rigid body and flexible generalized aerodynamic forces $\mathbf{P}_{b}^{\text {aero }}$ and $\mathbf{P}_{f}^{\text {aero }}$ that drive the nonlinear equations of motion (2).

The validation of the AIC based approach is done in the following way. First, the loads maneuver models are simulated in VarLoads using the implementation described in section V. Then, the individual time instances of the resulting trajectories are used as input to the panel method. Recall that the steady computations use a nonlinear relationship between the steady potential $\Phi_{S}$ and the resulting pressure and does not resort to a linearized version of the (unsteady) Bernoulli equation. Then the pressures from the quasisteady AIC approach and the direct panel method solution are compared on the basis of spanwise lift and moment distributions.

The computation with the steady panel method can not consider inherently unsteady effects, such as the downwash due to the modal velocities $\dot{\mathbf{u}}_{f}$. Since, the pressures in the AIC approach are recovered in a post processing step, such effects can be switched off, to allow a validation. I.e. while the trajectories are computed using the complete set of generalized AICs (15)-(14), for comparison of the spanwise lift distribution, individual effects of eq. (19) can be omitted.

Two test cases were selected, a trim case of a $2.5 \mathrm{~g}$ pull up manoeuvre and a transient roll manoeuvre. In both cases the model has to be trimmed with appropriate trim conditions. The transient roll manoeuvre is then simulated in time for 8 seconds.

The simulation speed of the AIC approach is very fast. It is possible to simulate these quasi-steady models faster than real time on an ordinary desktop computer. The panel method needs to evaluate a vector of 15000 pressures per flight state and is therefore considerably slower. Hence, for comparison, only every 10th time slice of the trajectory is evaluated.

\section{A. Symmetrical Pull-Up Manoeuvre}

The trim calculation of a symmetrical pull-up consists of a trim sequence of two consecutive trims. First the aircraft is trimmed for steady horizontal flight with the horizontal stabilizer. The second trim uses the pilot pitch command, i.e. the elevators to achieve the target load factor of $N_{z}=2.5 \mathrm{~g}$. This manoeuvre describes a circular path with a constant pitch rate, which can be approximated by $q_{c g}=\left(N_{z}-1\right) \cdot g / V$, where $q_{c g}$ is defined about the center of gravity.

The important trim results are summarized in table 1 .

It should be mentioned again that this is a trim of a fully flexible aircraft. The deformed model and the associated pressure distribution is shown in figure 1.

Due to the high load factor, a large deformation occurs which considerably alters the lift distribution compared to the rigid aircraft. The flexible twist at the tip nearly reaches 5 deg. Figure 2 depicts the spanwise bending and twist deformation along the loads reference axis of the right wing. 
Table 1. trim results for the $N_{z}=2.5 \mathrm{~g}$ pull-up manoeuvre

\begin{tabular}{rrr}
\hline trim variable & value & units \\
\hline$U_{\infty}$ & 167.79 & $\mathrm{~m} / \mathrm{s}$ \\
thrust & 611.285 & $\mathrm{kN}$ \\
angle of attack & 11.457 & $\mathrm{deg}$ \\
horizontal stabilizer & -3.9286 & $\mathrm{deg}$ \\
elevator & -5.8977 & $\mathrm{deg}$ \\
pitch rate & 5.0248 & $\mathrm{deg} / \mathrm{s}$ \\
\hline
\end{tabular}

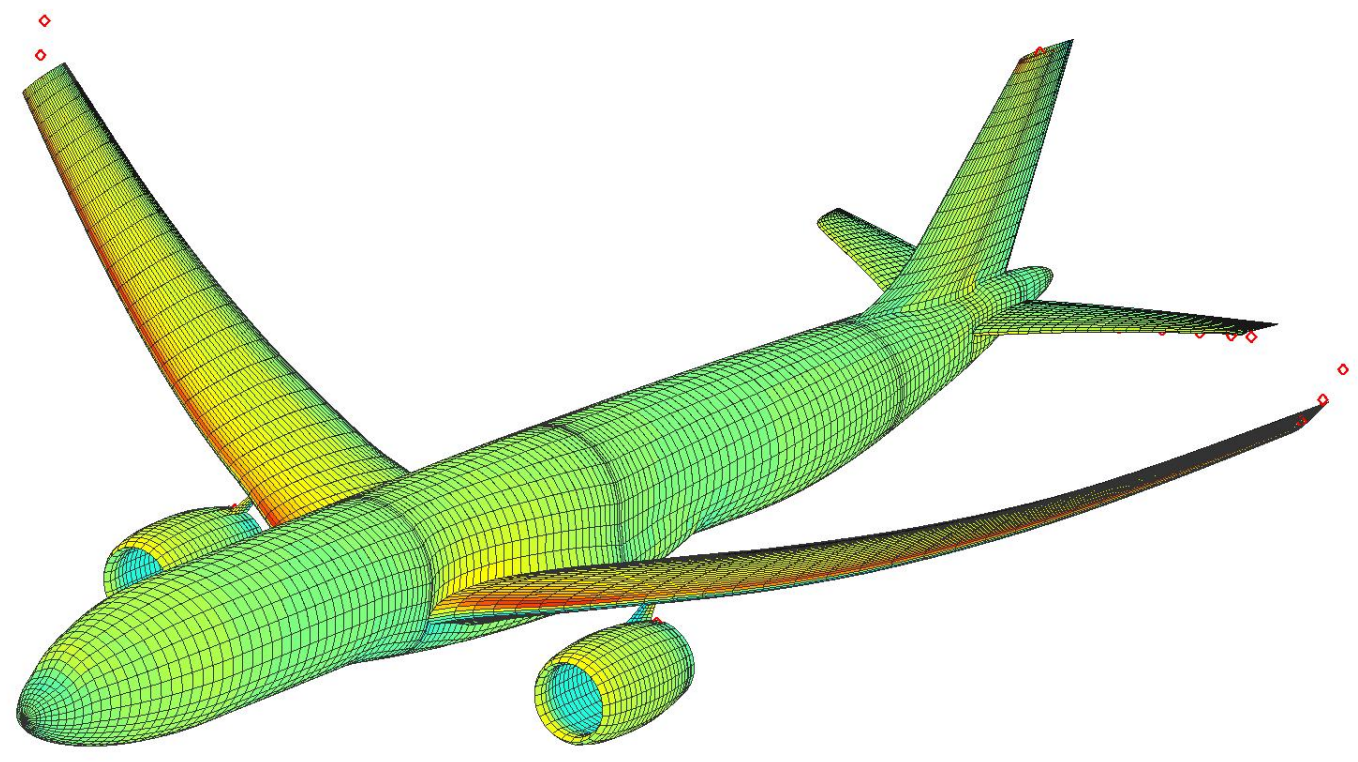

Figure 1. pressure distribution due to a $2.5 \mathrm{~g}$ pull-up maneuver using an AIC based 3D panel method approach.

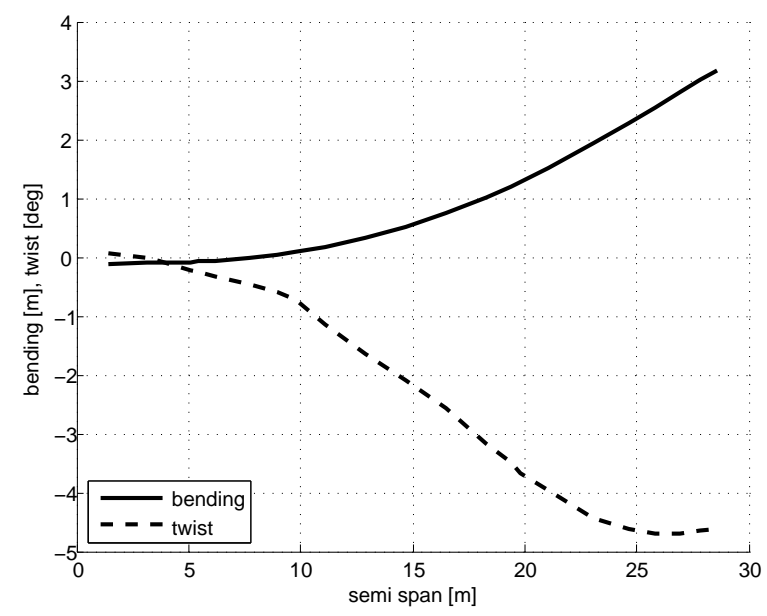

Figure 2. wing bending and twist for a $2.5 \mathrm{~g}$ vertical manoeuvre 
Figure 3 shows the spanwise lift distribution of the right wing for the AIC approach (red circles) and the Panel Method (black crosses). Further the lift distribution without the flexible increment is depicted with dashed lines. It should be noted that this does not represent a trim of the rigid aircraft, but merely shows the flexible effects on the lift distribution. A rigidly trimmed aircraft would have a much lower angle of attack, such that the integrated lift of the wing is roughly the same as in the flexible case.

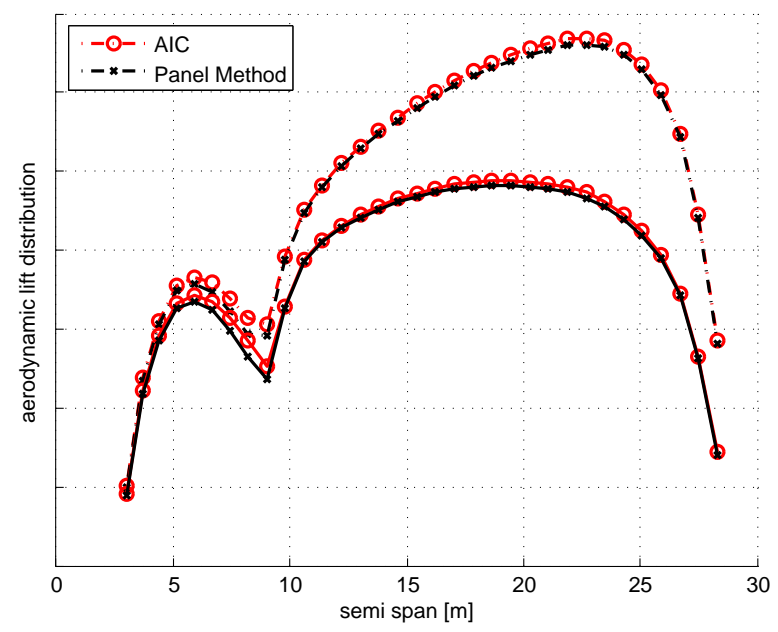

Figure 3. spanwise lift distribution, red for present AIC approach, black for Panel Method. Dashed lines are without flexible increment

The agreement between the results of the AIC approach and the Panel method is excellent. However this is also to be expected, since the resulting angle of attack is very close to one of the linearization points at $\alpha=10$ deg. Nevertheless this result is encouraging.

\section{B. Dynamic Roll Manoeuvre}

The second test case is a transient roll manoeuvre. An open loop model is used for the simulation, i.e. no flight control laws are implemented. The manoeuvre is starting from horizontal flight, then a step input to the pilot roll command deflects the ailerons and initiates the roll. The target is a steady roll rate of about $15 \mathrm{deg} / \mathrm{s}$. The maximum roll angle of roughly $\Phi=60 \mathrm{deg}$ is reached after 4 seconds. Then the sign of the roll command is reversed. Since the simulation model does not contain flight control laws, a rudder deflection is applied to keep the angle of sideslip low in order to yield a coordinated turn. These aggressive inputs may be unrealistic and even violate the quasi-steady assumption. However this way the structural dynamics of the aircraft is excited, emphasizing the validation aspects of the present method. The resulting trajectories of the simulation are show in figure 4.

When comparing the lift distribution, an issue with the control surfaces arises. In the steady computation of the panel method the normal vectors of the panels belonging to the control surface are rotated a finite angle, whereas in the AIC method, the linearized effect is simply scaled with the deflection angle. This leads to a significant overprediction of the control surface efficiency. Figure 5 shows the lift distributions of the wing for $\alpha=0$ deg and an aileron deflection of $\delta_{\text {ail }}=10 \mathrm{deg}$. Clearly, the AIC approach yields an overpredicted rolling moment. These issue are not at all uncommon in flight mechanics, where often nonlinear effects in the control surface deflections need to be considered. These issues are commonly handled by control surface efficiency look up tables to adapt to nonlinearities not only stemming from finite rotations but also other aerodynamic nonlinear effects. Therefore, for validation purpose, the control surface effects have been omitted and only the trajectory of the rigid body motion and the flexible deformation was computed when using the steady panel method. The AIC method accordingly sets the control surface vector $\mathbf{U}_{x 2}$ in equation (19) to zeros. Similarly, the effect of flexible motion due to $\dot{\mathbf{U}}_{f}$ needs to be omitted to allow for a meaningful comparison.

Figure 6 shows the rigid body forces and moments during the transient roll manoeuvre. The value of the force in $\mathrm{Z}$-direction is expressed in daN to allow for displaying all force components at the same scale.

The result of the AIC approach and the panel method compare very well. The forces in $\mathrm{y}$ and $\mathrm{z}$ direction are in very good agreement. For the values in $\mathrm{x}$ direction the agreement is not as good. The $\mathrm{x}$-force has a large component corresponding to the induced drag, which is not linear wrt. $\alpha$ and in this case is obtained by panel integration. It should be investigated if a Trefftz plane integration and a drag polar lookup improves the results. The moments are 

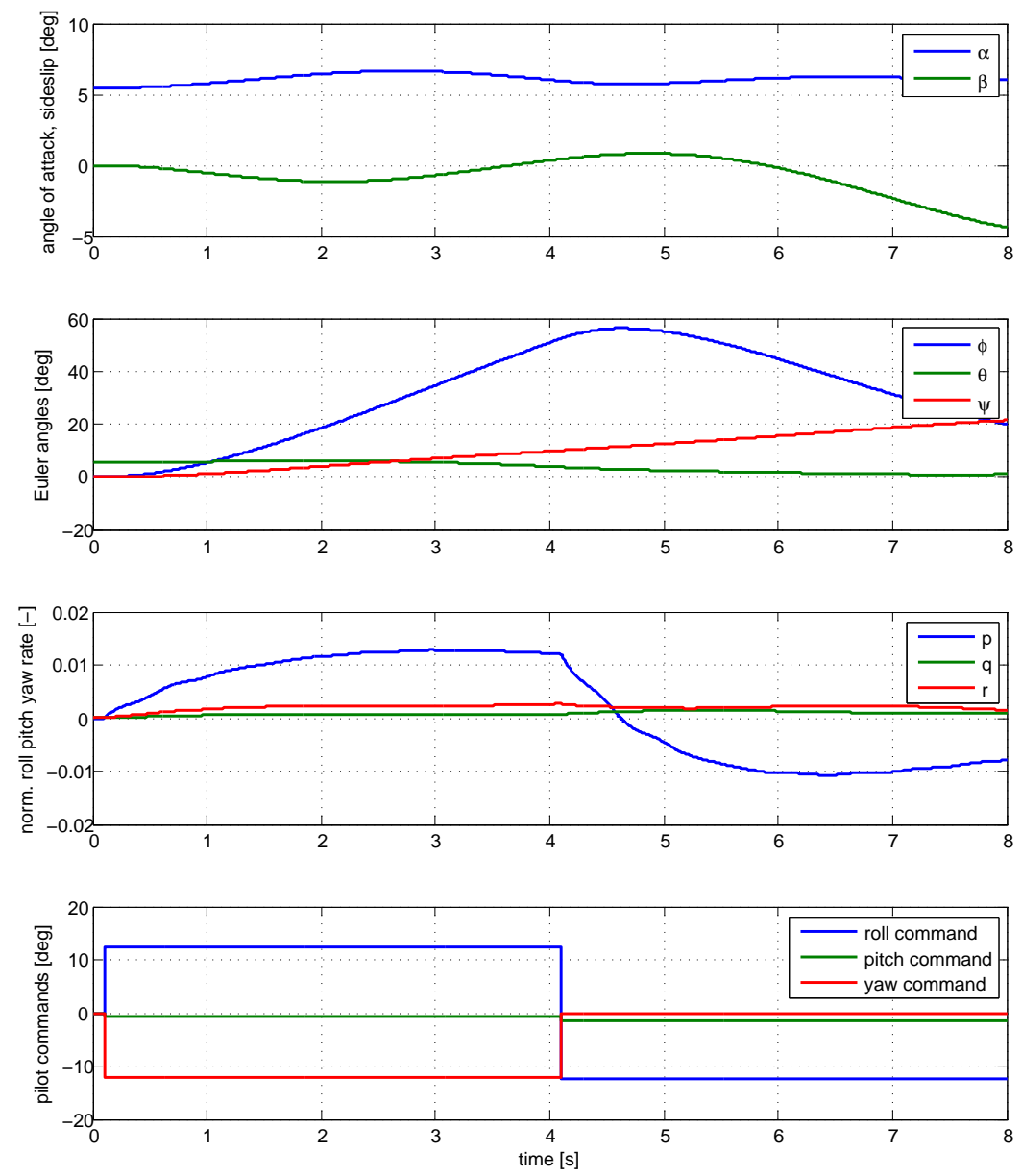

Figure 4. trajectories of the rolling manoeuvre

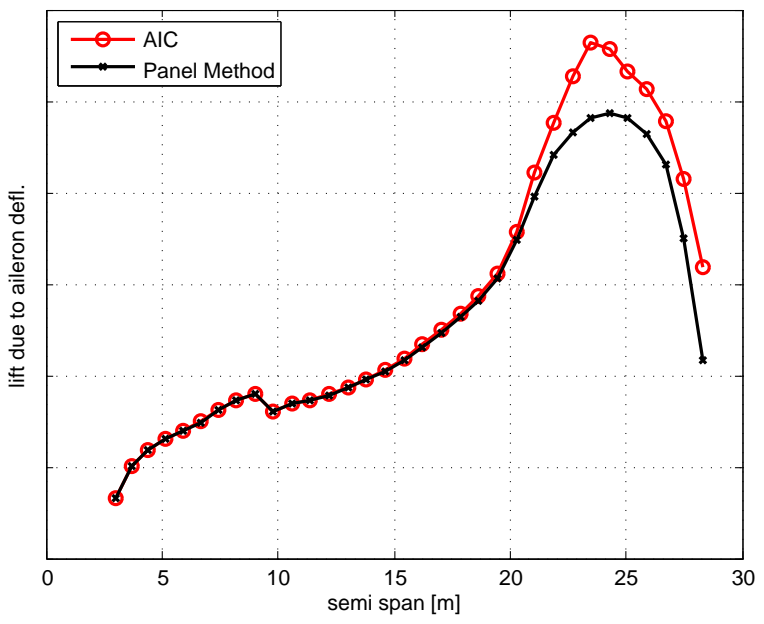

Figure 5. spanwise lift distribution due to 10 degree aileron deflection 

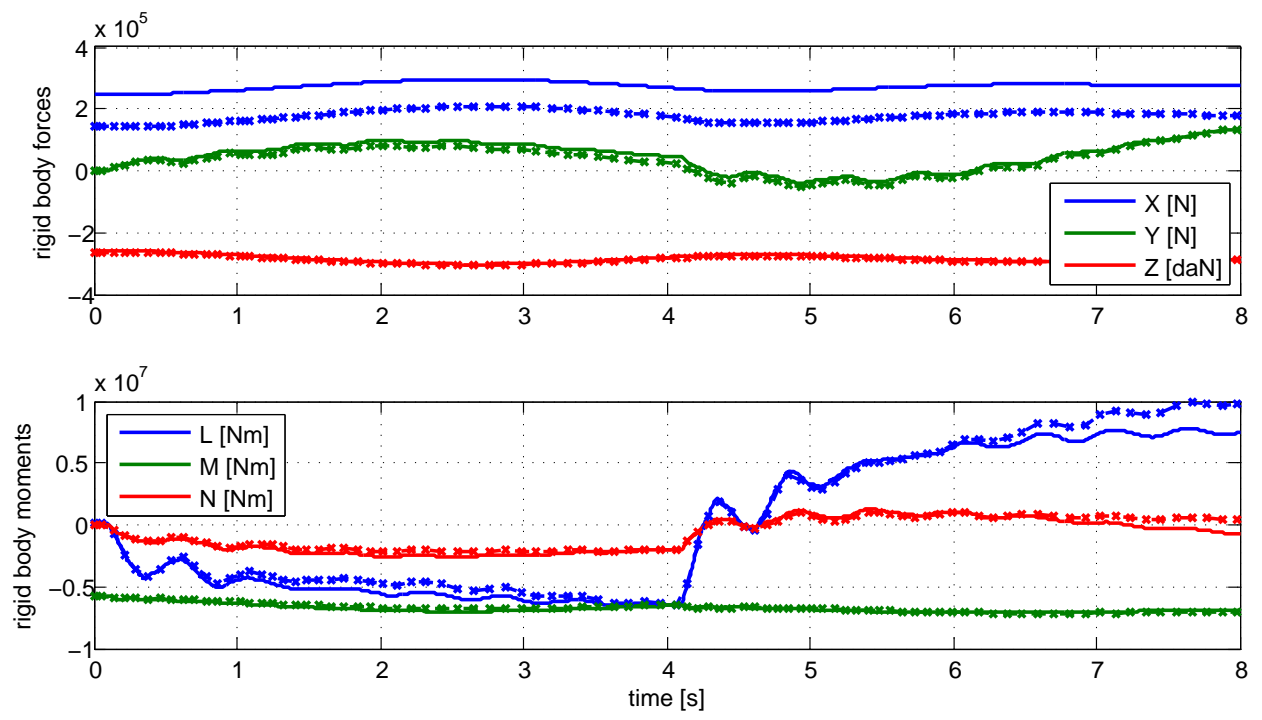

Figure 6. rigid body forces and moments for AIC (solid) and panel method (dashed). Force in $\mathrm{Z}$ expressed in daN.

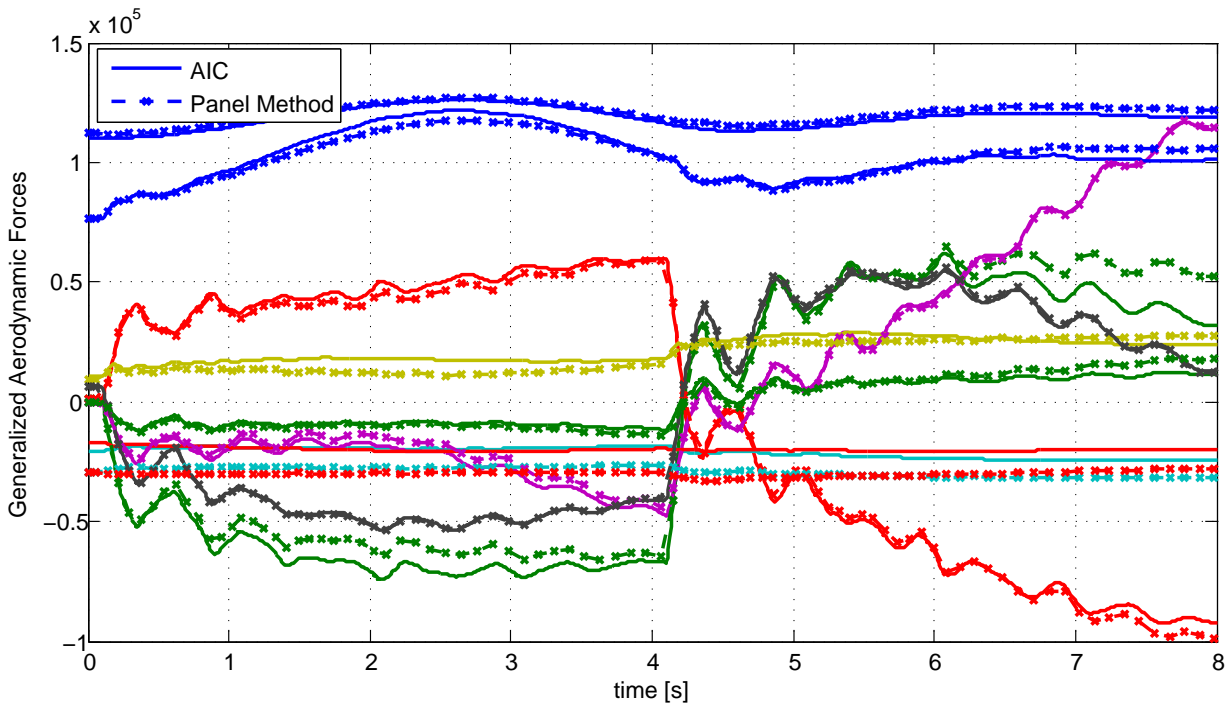

Figure 7. flexible generalized aerodynamic forces for AIC (solid) and panel method (dashed) 
also in good agreement. However, some differences in the rolling moment $L$ appear, which can probably be attributed to high roll rates leading to nonlinearities in the steady solution.

In figure 7 the generalized aerodynamic forces for the first ten modes are depicted. Again, although not perfect, the results compare well. The excitation of the structural dynamics in particular of the antisymmetrical modes can clearly be seen.

\section{Summary and Conclusion}

A successful demonstration of a proof of concept implementation using Aerodynamic Influence Coefficients from a 3D panel method in a loads analysis simulation has been presented. Compared to co-simulation schemes, the AIC approach allows a direct formulation of the model equations as a system of nonlinear ordinary differential equations. The fact that the AIC matrices can be generalized to a small size, reduces the computational cost and allows for very fast simulations. The pressures and loads can then be recovered in a postprocessing step. Further, the preparation of the full pressure AIC matrices happens in a preprocessing step and can be reused for different mass cases and manoeuvre load conditions. This allows for a quick determination of the loads envelope for aircraft design in a loop capable environment.

The quasi steady approximation using the AIC matrices at $k=0$ for manoeuver loads models was explained and justified. The requirement to account for nonlinearities depending on the angle of attack was met by implementing a simple linear interpolation scheme for the generalized AIC matrices.

The approach was successfully demonstrated on two test cases: a symmetrical high $N_{z}$ trim case and a transient roll manoeuvre. The results of the present AIC approach were compared to regular steady state computations using the 3D panel method at individual time instances of the trajectory. The spanwise lift and moment distributions of the wing agree excellently for the steady case as the trim angle of attack is very close to one of the linearization points. Also the results for the dynamic simulation compared well, however, with some caveats. The effects of the control surfaces were overpredicted due to the simple linear scaling of the small perturbation results.

The remaining discrepancies can be explained with simplifications and modeling differences in the current implementation. The motion induced body terms of equation (13) are currently neglected. It should be investigated how much influence these terms have on the results. The linear interpolation of the AICs between only two angles of attack is very crude and might lead to poor results far away from the linearization points. An improved interpolation scheme covering also other nonlinear parameters is already envisaged. The overprediction of effects due to large control surface deflections, instead of linearly scaled perturbations, could be handled by efficiency look-up tables.

The results using the AICs from a 3D panel method for manoeuver loads simulations are very encouraging, and the accuracy of the aerodynamic load prediction can probably be even further increased when implementing the suggested improvements.

\section{Acknowledgements}

The author wishes to thank Steve Fiddes and Chris Burkett from Flow Solutions Limited for the continuous NEWPAN support and helpful discussions on panel methods. Special thanks go the DLR colleague Martin Leitner for preparing the panel model used in this paper.

\section{References}

[1] Hedman, S., "Vortex Lattice Method for Calculation of Quasi Steady State Loadings on Thin Elastic Wings," Tech. Rep. Report 105, Aeronautical Research Institute of Sweden, October 1965.

[2] Albano, E. and Rodden, W., "A Doublet-Lattice Method for Calculating Lift Distributions on Oscillating Surfaces in Subsonic Flows," AIAA Journal, Vol. 7, No. 2, 1969, pp. 279-285.

[3] W.P. Rodden, J.P. Giesing and T.P. Kalman, "New Developments and Applications of the Subsonic Doublet-Lattice Method for Nonplanar Configurations," AGARD Symposium on unsteady aerodynamics for aeroelastic analyses of interfering surfaces, No. AGARD-CP-80-71, AGARD, 1971.

[4] W.P. Rodden, P.F. Taylor and S.C. McIntosh Jr., "Further Refinement of the Subsonic Doublet-Lattice Method," Journal of Aircraft, Vol. 9, No. 10, 1998, pp. 693-702.

[5] Flow Solutions Ltd., "NEWPAN," http://www.flowsol.co.uk/products/newpan/.

[6] A. Schütte,G. Einarsson, A. Raichle, B. Schöning, M. Orlt, J.Neumann, J. Arnold, W. Mönnich und T. Forkert, "Numerical Simulation of Maneuvering Aircraft by Aerodynamic, Flight Mechanics and Structural Mechanics Coupling," 45th AIAA Aerospace Sciences Meeting and Exhibit, Reno, Nevada, January 8-11, No. AIAA 2007-1070, AIAA, January 2007. 
[7] Ritter, M. and Dillinger, J., "Nonlinear Numerical Flight Dynamics for the Prediction of Maneuver Loads," International Forum on Aeroelasticity and Structural Dynamics, No. IFASD-2011-143, 2011.

[8] Christopher J. Sequeira, David J. Willis, and Jaime Peraire, "Comparing Aerodynamic Models for Numerical Simulation of Dynamics and Control of Aircraft," 44th AIAA Aerospace Sciences Meeting and Exhibit, No. AIAA 2006-1254, 2006.

[9] J. Katz and A. Plotkin, Low Speed Aerodynamics: From Wing Theory to Panel Methods, McGraw-Hill, 1991.

[10] Roger, K. L., "Airplane Math Modeling Methods for Active Control Design," AGARD Structures and Materials Panel, No. AGARD/CP-228, AGARD, April 1977, pp. 4-1 - 4-11.

[11] Kier, T. and Looye, G., "Unifying Manoeuvre and Gust Loads Analysis," International Forum on Aeroelasticity and Structural Dynamics, No. IFASD-2009-106, 2009.

[12] R. L. Bisplinghoff, H. Ashley, R. L. Halfman, Aeroelasticity, Dover Publications Inc., 1955.

[13] J. Hofstee, T. Kier, C. Cerulli, G. Looye, "A Variable, Fully Flexible Dynamic Response Tool for Special Investigations (VarLoads)," International Forum on Aeroelasticity and Structural Dynamics, 2003.

[14] T. Kier, G. Looye and J. Hofstee, "Development of Aircraft Flight Loads Analysis Models with Uncertainties for Pre-Design Studies," International Forum on Aeroelasticity and Structural Dynamics, June 2005.

[15] Kier, T. M., "Comparison of Unsteady Aerodynamic Modelling Methodologies with respect to Flight Loads Analysis," AIAA Atmospheric Flight Mechanics Conference, 15-18 August, San Fancisco, CA, USA, No. AIAA 2005-6027, AIAA, 2005.

[16] T. Kier, G. Looye, M. Scharpenberg, and M. Reijerkerk, "Process, Methods and Tools for Flexible Aircraft Flight Dynamics Model Integration,” International Forum on Aeroelasticity and Structural Dynamics, No. IF-060, CEAS/AIAA, 2007.

[17] Kier, T., "An Integrated Loads Analysis Model including Unsteady Aerodynamic Effects for Position and Attitude dependent Gust Fields," International Forum on Aeroelasticity and Structural Dynamics, No. IFASD-2011-052, 2011.

[18] Kier, T., “An Integrated Loads Analysis Model for Wake Vortex Encounters," International Forum on Aeroelasticity and Structural Dynamics, No. IFASD-2013-30C, 2013.

[19] R. J. Guyan, "Reduction of stiffness and mass matrices,” Journal of Aircraft, Vol. 3, No. 2, 1965, pp. 380.

[20] M. R. Waszak and D. K. Schmidt, “On the Flight Dynamics of Aeroelastic Vehicles," AIAA Atmospheric Flight Mechanics Conference, No. AIAA 86-2077, AIAA, 1986, pp. 120-133.

[21] M. R. Waszak and D. K. Schmidt, "Flight Dynamics of Aeroelastic Vehicles," Journal of Aircraft, Vol. 25, No. 6, 1988, pp. 563-571.

[22] M. R. Waszak, C. S. Buttrill and D. K. Schmidt, "Modeling and Model Simplification of Aeroelastic Vehicles: An Overview," Tech. Rep. NASA TM-107691, NASA LARC, 1992.

[23] C. Reschke, Integrated Flight Loads Modelling and Analysis for Flexible Transport Aircraft, Ph.D. thesis, Universität Stuttgart, 2006.

[24] Pistolesi, E., "Betrachtungen über die gegenseitige Beeinflussung von Tragflügelsystemen," Gesammelte Vorträge der Hauptversammlung 1937 der Lilienthal Gesellschaft, 1937.

[25] Harder, R. and Desmarais, R., "Interpolation Using Surface Splines,” Journal of Aircraft, Vol. 9, No. 2, 1972, pp. $189-191$.

[26] Rodden, W. P. and Johnson, E. H., MSC.Nastran Aeroelastic Analysis User's Guide, MSC, 1994.

[27] Göthert, B., "Ebene und räumliche Strömung bei hohen Unterschallgeschwindigkeiten: Erweiterung der Prandtl'schen Regel," Tech. Rep. Bericht 127, Lilienthal Gessellschaft, 1940.

[28] Theodorsen, T., "General Theory of Aerodynamic Instability and the Mechanism of Flutter," Tech. Rep. NACA Report 496, NACA, 1938.

[29] European Aviation Safety Agency, Certification Specifications for Large Aeroplanes CS-25, Vol. Subpart C - Structure, EASA, 2010. 